\title{
Commissioning of the NDDL-40 Micro-Channel Plate Neutron Detector System at Oregon State University
}

\author{
Nicholas M. Boulton ${ }^{1, a^{*}}$, Steven R. Reese ${ }^{2, b}$, and Aaron E. Craft ${ }^{1, c}$ \\ ${ }^{1}$ Idaho National Laboratory, PO Box 1625, MS 2211, Idaho Falls, ID 83415, USA \\ ${ }^{2}$ Radiation center, 3451 Jefferson Way, Corvallis, OR 97330, USA \\ aNicholas.Boulton@inl.gov, ${ }^{\mathrm{b}}$ Steven.Reese@oregonstate.edu, ${ }^{\mathrm{c}}$ Aaron.Craft@inl.gov
}

\section{Keywords: Neutron Radiography, Neutron Tomography, Micro-Channel Plate}

\begin{abstract}
The Neutron Radiography Facility (NRF) at Oregon State University (OSU) has been modified to begin working on the non-destructive evaluation of concrete materials to study the early stages of shrinkage, cracking, and water transport of concrete during the curing process. The objective of this work was to investigate the efficiency and spatial resolution of the NDDL 40 micro-channel plate (MCP) detector for the use of neutron radiography and tomography to determine its applicability for examining concrete. Working in collaboration with the School of Civil and Construction Engineering, the NRF at OSU has added a NDDL-40 vacuum-sealed neutron imaging detection system with a delay line system readout developed by NOVA Scientific. This study found that the system installed at the NRF was capable of a maximum spatial resolution of $\sim 250 \mu \mathrm{m}$ with a neutron detection efficiency of $5.49 \%$. Significant artifacts from the detector system and image noise degraded the quality of the tomographic reconstruction to such an extent that this neutron imaging system could not be used to visualize the desired phenomena in concrete.
\end{abstract}

\section{Introduction}

Oregon State University acquired a NDDL-40 detector system from NOVA Scientific. for examination of water transport through concrete. The detector was evaluated through a series of measurements at the Oregon State TRIGA Reactor (OSTR) to determine special resolution and detector efficiency. Similar testing has been performed previously using an earlier MCP system [1]. The detector was advertised to provide spatial resolution less than $50 \mu \mathrm{m}$. The vacuumsealed detector contained two neutron sensitive micro-channel plates with a delay line anode output. The MCP's are $1 \mathrm{~mm}$ thick glass plates with millions of 4-11 $\mu \mathrm{m}$ diameter channels in a hexagonal array through each plate [2]. These micro-channel plates are doped with boron, because of its large thermal neutron absorption cross-section. A neutron absorbed by the ${ }^{10} \mathrm{~B}$ undergoes a ${ }^{10} \mathrm{~B}(\mathrm{n}, \alpha) \mathrm{Li}^{7}$ interaction, producing daughter products that interact with the glass, creating secondary electrons. A voltage potential across the plates allows the secondary electrons to create an electron cascade with a net signal gain of approximately $10^{7}$. This creates a detectable pulse from the anode which is registered by the detector as a count.

MCP detector systems have been used extensively for neutron imaging of dynamic phenomena due to their exceptionally fast dynamic response [3]. The detector response of some MCP neutron imaging systems is so fast that it can be used with pulsed neutron sources for timeof-flight (TOF) measurements, enabling a wide range of scientific applications. An MCP system was used to perform TOF resonance absorption measurements at LANSCE to visualize the distribution of ${ }^{235} U$ and ${ }^{238} U$ in nuclear fuel pellets [4]. Another benefit of MCP's is their lower gamma-ray sensitivity relative to many other neutron imaging technologies. MCP have been demonstrated to be able to directly examine highly radioactive irradiated nuclear fuel $[5,6]$. 


\section{Reference Standard Test Objects}

ASTM E545 [7], describes a method for determining image quality of a thermal neutron beam that is specifically used for radiography. The standard describes the use two different test objects. The first standard test object is called the Beam Purity Indicator (BPI), which is constructed as a polytetrafluoroethylene block containing two boron nitride disks, two lead disks, and two cadmium strips [7]. This standard provides a method for determining the thermal neutron content and gamma content measures image sharpness and overall quality of direct neutron radiography with gadolinium conversion screens with film and is not directly applicable to digital neutron radiography systems. The second indicator used is the Sensitivity Indicator (SI). This indicator qualitatively determines the sensitivity of the neutron radiograph by observing holes and gaps of known dimensions between each shim. While not directly applicable to digital neutron radiography systems, the standard [7] can be applied to the extent applicable to determine the category of the facility. Another image quality indicator used was a cadmium strip provided by NOVA Scientific was imaged to obtain additional data on the detectors image resolution and sharpness. The strip was a $0.050 \mathrm{~cm}$ thick cadmium strip with a series of holes along the center line. The smallest holes are $250 \mu \mathrm{m}$ diameter with a separation of $250 \mu \mathrm{m}$ between holes.

\section{Measurement of the Neutron Beam Flux}

Before determining the detector efficiency, the thermal neutron flux needed to be measured. This was performed using gold activation foils. The gold foil is activated and decays concurrently, and the activity can be determined at any point in time using the differential equation, $\mathrm{dN} / \mathrm{dt}=\mathrm{R}$ $\lambda \mathrm{N}$, where $\mathrm{dN} / \mathrm{dt}$, the rate of change in radioactive nuclei over time, is the difference between the rate of activation, $R$, and the activity of the sample at a point in time, $A(t)=\lambda N(t)$, which is the product of the decay constant, $\lambda$, and the number of radioactive nuclei, $N(t)$. Thus, the activity at a point in time, is given by:

$$
A(t)=\mathrm{N}(\mathrm{t}) \lambda=\mathrm{R}\left(1-\mathrm{e}^{-\lambda t_{0}}\right)
$$

After a 7-hour beam exposure time with the OSTR power at $1 \mathrm{MW}$, the activity of each of the two foils was measured using a calibrated HPGe detector. Because the foils are continually decaying after irradiation, the time between each step was accounted for through the following equation:

$$
A=\frac{\lambda(\mathrm{C}-\mathrm{B})}{\varepsilon\left(1-e^{-\lambda t_{0}}\right)\left(e^{-\lambda t_{1}}-e^{-\lambda t_{2}}\right)}, \#(2)
$$

where $\mathrm{C}$ is the counts measured from the detector, $\mathrm{B}$ is the number of background counts expected in the counting time, $t_{0}$ is the time of irradiation, $t_{1}$ is the time of the foils removal from the neutron flux, $t_{2}$ is the time the foils are counted by the HPGe detector, and $\varepsilon$ is the counting efficiency of the detector. Each gold foil was of the same purity, size and thickness. For the measurement, one bare foil was placed in front of the beam while the other foil was placed in a $\mathrm{Cd}$ cover in the beam. The difference in radioactivity between the two foils can be used to determine the flux from thermal and higher-energy neutrons. By subtracting the amount of activation of the $\mathrm{Cd}$ covered gold foil from the bare gold foil the activity from only thermal neutrons can be calculated.

Gold has an average thermal absorption cross-section of 98.65 barns. After neutron absorption, the ${ }^{197} \mathrm{Au}$ (100\% natural abundance) becomes ${ }^{198} \mathrm{Au}$ which has a half-life of 2.7 days and decays via beta emission to become ${ }^{198 \mathrm{~m}} \mathrm{Hg}$ that promptly decays into stable ${ }^{198} \mathrm{Hg}$ via emission of a $411 \mathrm{keV}$ gamma-ray. Thermal neutron flux was obtained from the activities 
measured with bare and Cd-covered foils given material constants, decay time and irradiation time, as shown in Eq. 3.

$$
A=\left(\phi_{t h} \sigma_{t h}+\phi_{\text {epi }} \sigma_{\text {epi }}\right) * N\left(1-e^{-\lambda t}\right)
$$

Solving for $\phi_{t h}$ gives,

$$
\phi_{t h}=\left(\frac{A_{A u}}{N_{A u}\left(1-e^{-\lambda t}\right)}-\left(\phi_{e p i} * \sigma_{e p i}\right)\right) /\left(\sqrt{\frac{\pi}{2}} * \sigma_{t h}\right) . \#(4)
$$

Table 1 shows values used to calculate the thermal neutron flux of OSTR's Beam Port 3, which was determined to be $9.42 \times 10^{5} \pm 1.55 \times 10^{4} \mathrm{n} / \mathrm{cm}^{2} / \mathrm{s}$ with the reactor power at $1 \mathrm{MW}$.

Table 1. Measurements used for Gold foil experiment.

\begin{tabular}{|lc|}
\hline Variable & Value \\
\hline Irradiation time [hr] & 6.96 \\
$\lambda_{\mathrm{Au}-198}\left[\mathrm{hr}^{-1}\right.$ ] & 6.17 \\
$\mathrm{~N}_{\mathrm{Au}-197}$, bare gold [atoms] & $2.65 \times 10^{20}$ \\
$\mathrm{~N}_{\mathrm{Au}-197}$, Cd-covered [atoms] & $2.63 \times 10^{20}$ \\
$\mathrm{Thermal} \mathrm{cross} \mathrm{section} \mathrm{[cm}^{2}$ ] & $9.87 \times 10^{-23}$ \\
Epithermal cross-section $\left(\mathrm{cm}^{2}\right]$ & $1.55 \times 10^{-21}$ \\
Activity, bare [Bq] & $3.17 \times 10^{3}$ \\
Error of activity, bare [Bq] & $5.20 \times 10^{1}$ \\
Activity, Cd-covered [Bq] & $9.34 \times 10^{2}$ \\
Error of activity, Cd-covered [Bq] & $1.53 \times 10^{1}$ \\
\hline
\end{tabular}

\section{Neutron Detection Efficiency}

The rate of neutrons incident on the detector must be calculated from the neutron flux and the number of neutrons registered must be measured by the detector. The neutron flux $\left(9.4242 \times 10^{5}\right.$ $\mathrm{n} / \mathrm{cm}^{2} / \mathrm{s}$ ) multiplied by the area of the $2 \mathrm{~cm}$ diameter detector gives the rate of neutrons incident on the detector as $1.184 \times 10^{7} \mathrm{n} / \mathrm{s}$. The number of neutron counts registered was counted by the detector software during exposure, which was $6.5 \times 10^{5} \mathrm{n} / \mathrm{s}$. The total efficiency is then the ratio of the two, giving an approximate detector efficiency of $5.49 \%$.

\section{Image Processing of Neutron Radiographs}

After image acquisition, image post-processing optimizes sharpness and contrast to clean up each image. Issues that can reduce the quality of the image include noise from the detector, foreign objects in the field of view, ghosting, or non-uniform image acquisition parameters. Images were processed after each exposure using ImageJ image processing software [8]. For most corrections, a flat-field image (i.e. background and/or open beam image), is taken before each radiograph or series of radiographs with the object of interest positioned outside the field of view. During post processing the flat-field is divided into the raw image containing the desired object to be viewed. Flat-field normalization corrects for variations of the detector response and beam non-uniformity. The software uses the following equation to normalize the intensities across the image:

$$
I_{1}=\left(\frac{i_{1}}{i_{2}}\right) * k_{1}+k_{2}
$$

where $I_{1}$ is the new image after processing, $i_{1}$ is the image with the object, $i_{2}$ is the flat-field image, $k_{1}$ is the average intensity in $i_{1}$, and $k_{2}$ is a minimum intensity which was always 0 . 
Multiplying by the $k_{1}$ scales the grayscale values to more completely fill the histogram per the available bit-depth of the final image. The image may need to be further processed by adjusting the contrast or removing noise to better visualize features of interest. Fig. 1 shows the process at which the radiographs are processed and corrected. Figs. 1a and 1b show the flat field and uncorrected radiograph of the BPI, respectively. Flat field correction was applied to the image of the BPI using Eq. 5, resulting in the image shown in Fig. 1c. The result shows the removal of detector noise and high intensities that are in the radiograph that are not part of what is being radiographed. The resulting image was then processed by normalizing the histogram and enhancing contrast, which yields the image of the BPI shown in Fig. 1d.
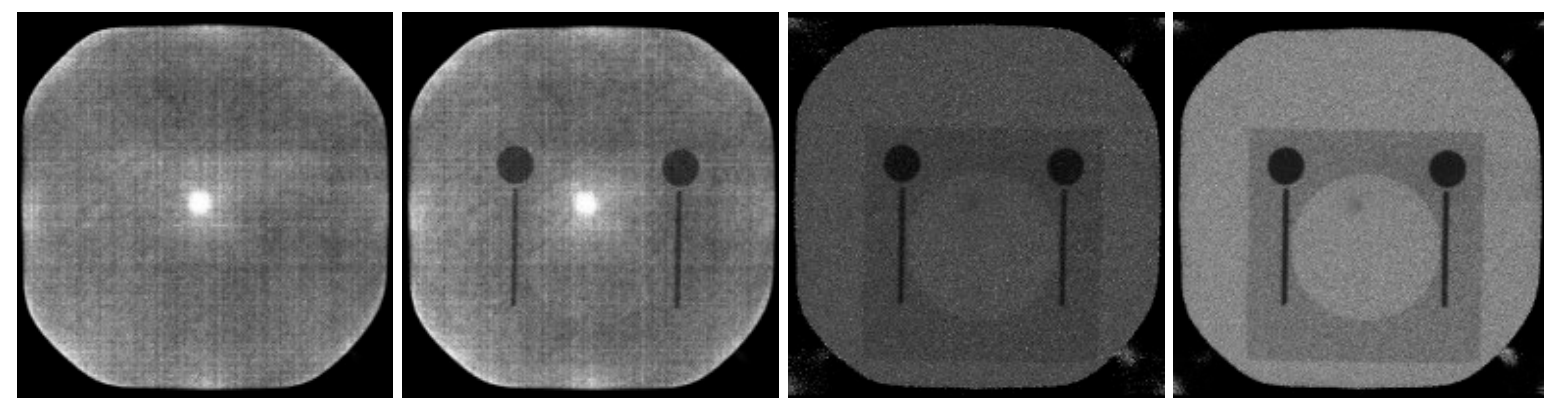

Fig. 1. Resulting radiographs and processed images of a BPI. a) Flat-field image of the beam. b) Radiograph of the BPI. c) Processed radiograph. d) Processed radiograph with enhanced contrast.

\section{Neutron Tomography}

For tomography reconstruction, the post processing is similar to radiography image processing, but the process is applied to multiple images. This is done by placing the desired object on a motorized stage to precisely rotate the object. A $500 \mathrm{~mm}$ linear travel (IMS500PP) and $360^{\circ}$ rotational (URS75BPP) stage were acquired from the Newport Corporation. Newport's proprietary software allows stages to be controlled remotely from outside the NRF. This allows the desired object to be moved horizontally and rotated while keeping the shutter for the beam open. For adequate tomography, radiography images should be acquired over a minimum range of $180^{\circ}$. A set of 180 radiographs were taken using the maximum field of view of $4 \mathrm{~cm}$ and L/D of 100 .

The minimum distance between the center of rotation and the detector based on the dimensions of the BPI is $13.125 \mathrm{~mm}$ (the minimum distance from the center to the outside edges of the $25 \times 25 \times 8 \mathrm{~mm} \mathrm{BPI}$ ) plus the $\sim 12.5 \mathrm{~mm}$ distance from the detector to the vacuum housing window, for a total minimum object-to-detector distance of $25.625 \mathrm{~mm}$. Thus, the minimum geometric unsharpness for $\mathrm{L} / \mathrm{D}$ of 100 would be $256.25 \mu \mathrm{m}$ when performing neutron tomography. The distance from the vacuum housing window to the detector would add to the object-detector distance, further increasing geometric unsharpness.

A macro was written in ImageJ to process the images automatically, applying a flat-field correction and enhancing contrast for each image. After processing projections with ImageJ, Octopus reconstruction software was used to perform tomographic reconstruction of the images [9]. Using Octopus, the processed images from ImageJ were first cropped to the field of view of the object. Even though the setup for the experiment could be assumed to be a parallel beam, the mode set in Octopus was set to cone beam with the appropriate source-to-object distance and source-to-detector distance for the experiment. The software can adjust filters to remove more noise from each projection. A bi-linear interpolation filter characterized each pixel and voxel to 1 $\mu \mathrm{m}$. Sinograms are produced from the filtered projections using filtered back-projection. 


\section{Results \& Discussion}

Fig. 2a and 2b show the processed radiographs of the SI and BPI, respectively. For radiographs, the objects were placed directly on the detector window to reduce geometric unsharpness. Considering the radiograph of the SI in Fig. 2a, there are no visible holes (i.e. does not meet any category requirements) but five visible shims (category IV for this metric). The metrics derived from the BPI in Fig. 2b are not applicable for digital neutron radiography systems, so they cannot be used in determining the ASTM facility category. Examination of Fig. 2c shows the radiograph of a cadmium strip used to help quantify the spatial resolution. The effective spatial resolution of the detection system was found to be in the range of $\sim 250 \mu \mathrm{m}$, which is the diameter of the smallest holes in the cadmium strip with a known spacing of $250 \mu \mathrm{m}$ between each hole. The samples being placed on the window of the vacuum housing would give an object-to-detector distance of $\sim 12.5 \mathrm{~mm}$, so the resulting geometric unsharpness with an L/D of 100 would be 125 $\mu \mathrm{m}$, which may have limited the spatial resolution obtained in these measurements.
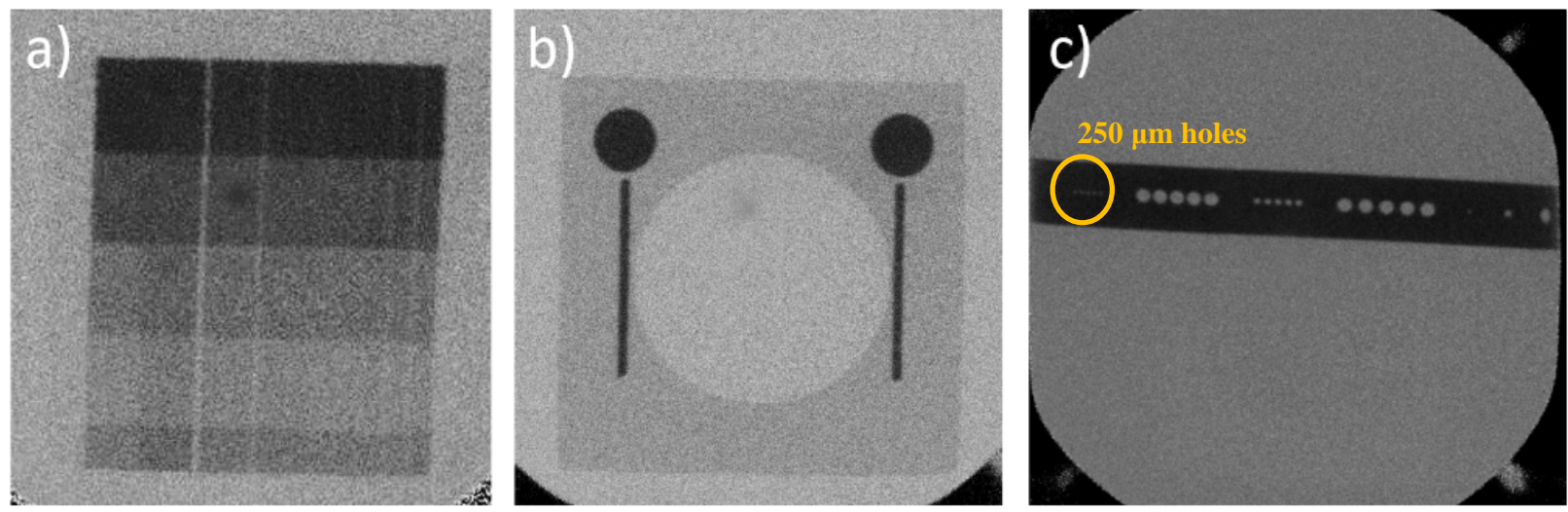

Fig. 2. a) Neutron radiographs of the SI and b) BPI (right). c) Image of a cadmium strip with various size holes.

Fig. 3 shows a representative radiograph, sinograms from multiple projections, and the resulting tomographic reconstruction. While the resulting tomographic reconstruction visibly shows the major features of the BPI (e.g. cadmium wires, boron nitride discs), artifacts from inconsistent detector performance and the significant amount of noise in each image yielded poor overall quality of the resulting reconstruction. While the boron discs and cadmium wires rendered very well, the polyethylene block is hardly visible.
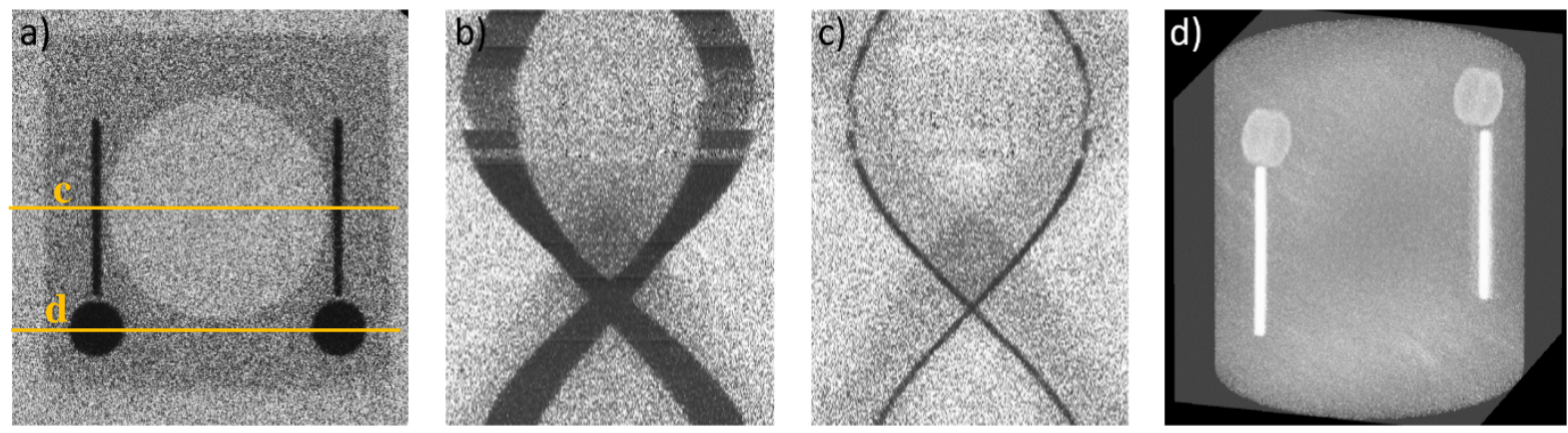

Fig. 3. a) Radiograph of a BPI. b\&c) Resulting sinograms. d) Isomeric view of the resulting tomographic reconstruction. 
Tomographic reconstruction was done using the Octopus software. Fig. 3 shows a pair of sinograms that were rendered using radiographs of the BPI. The left sinogram follows the boron disc of the BPI while the sinogram on the right shows the path the cadmium wires within the BPI. Inconsistencies are visible in the sinograms that can be attributed to periodic, temporary, and unexplained degradation of contrast signal to noise ratio of the NDDL40 detector system.

\section{Conclusion}

The objective of this work was to investigate efficiency and spatial resolution of the NDDL40 MCP vacuum sealed detector from NOVA Scientific for the use of neutron radiography and tomography. The detector is based on using borated micro channel glass plates to detect incoming thermal neutrons through the ${ }^{10} \mathrm{~B}(\mathrm{n}, \alpha)^{7} \mathrm{Li}$ interaction. The NDDL $40 \mathrm{MCP}$ detector has been shown to be capable of producing neutron radiographs and tomography. Radiographs exhibited inconsistent image quality due to the varying background, low signal to noise ratio, and low detector efficiency. This led to issues in rendering quality tomographic reconstructions. More than half of the 190 projections taken for tomography had to be reacquired. The NDDL-40 MCP detector installed at the OSTR Beam Port 3 demonstrated a neutron detection efficiency of $5.49 \%$ with a spatial resolution of approximately $250 \mu \mathrm{m}$.

\section{References}

[1] W.J. Williams, "Neutron Radiography and Tomography: Determining and Optimizing Resolution of Neutron Sensitive Multi Channel Plate Detectors," Corvallis, 2013.

[2] O. H. Siegmund, J. V. Vallerga, A.S. Tremsin, J. Mcphate and B. Feller, "High Spatial Resolution Neutron Sensing Mincochannel Plate Detectors," Nuclear Instruments and Methods in Physics Research A 576, 178-182, 2007. https://doi.org/10.1016/j.nima.2007.01.148

[3] C.D. Ertley, O.H.W. Siegmund, J. Hull, A. Tremsin, A. O’Mahony, C.A. Craven, and M.J. Minot, "Microchannel Plate Imaging Detectors for High Dynamic Range Applications," IEEE Trans. Nuc. Sci. 64(7) 1774-1780, 2017. https://doi.org/10.1109/TNS.2017.2652222

[4] A.S. Tremsin, S.C. Vogel, M. Mocko, M A M Bourke, V. Yuan, R.O. Nelson, D.W. Brown, and W.B. Feller, "Non-destructive studies of fuel pellets by neutron resonance absorption radiography and thermal neutron radiography," Nuc. Mat. 440, 633-646, 2013. https://doi.org/10.1016/j.jnucmat.2013.06.007

[5] Tremsin, A.S., Craft, A.E., G.C. Papaioannou, et al., “On the possibility to investigate irradiated fuel pins nondestructively by digital neutron radiography with a neutron-sensitive microchannel plate detector with Timepix readout,” Nucl. Instr. Meth. in Physics Research A, 927, 109-118, 2019. https://doi.org/10.1016/j.nima.2019.02.012

[6] Tremsin, A.S., Craft, A.E., A.M.M. Bourke, et al., 2018. Digital neutron and gamma-ray radiography in high radiation environments with an MCP/Timepix detector. Nucl. Instr. Meth. in Physics Research A 902, 110-116, 2018. https://doi.org/10.1016/j.nima.2018.05.069

[7] ASTM E545-15, "Standard Test Method for Determining Image quality in Direct Thermal Neutron Radiographic Examination," ASTM International, West Conshohocken, PA, 2014.

[8] W. Rasband, "ImageJ," 1997. [Online]. Available: http://imagej.nih.gov.

[9] Octopus Imaging, Octopus Reconstruction User Manual, Ghent, 2016. 